\title{
LA MÚSICA COMO CIENCIA \\ EN LOS TEÓRICOS ESPAÑOLES DEL RENACIMIENTO: JUAN BERMUDO (1555) Y FRANCISCO SALINAS (1577)
}

Paloma OTAOLA

\begin{abstract}
It is usual to show an opposition between the theory and practice, musicus and cantor in the music of Middle-Ages whose origin lies in the writings of St Augustine and Boethius. The superiority of Music Theory as a rational science influences the reject of the hearing in musical judgment. In the same way, the performer and the singer are considered inferior to the musician, id est. who has the scientific knowledge of the music. The legacy of this tradition is present in several treatises as well the opposition between the musician (the theorist) and the performer, singer or composer (practician). However, the real aim of Juan Bermudo and Francisco Salinas, is not to reject practice music, as inferior, but to promote the need of a scientific knowledge to well practice music. The wish of both theorits, as we can read in their writings, is to combat the ignorance of performers and singers which difficult the music development and growing. We can see the influence of the aristotelism in the importance of the union of theory and practice raising from experience. Both writers, Bermudo and Salinas, proclaim the need of the hearing in musical judgment.
\end{abstract}

\section{Resumen}

Tradicionalmente se suele presentar una oposición entre música teórica y práctica, entre musicus y cantor, de origen pitagórico-platónico, transmitida a la Edad Media por San Agustín y Boecio. La superioridad de la música teórica, concebida como ciencia racional lleva a rechazar el papel del oído en el juicio musical. Así mismo, el instrumentista o el cantor es considerado inferior al que posee el conocimiento racional de la música, esto es al músico teórico. El peso de la tradición en el Renacimiento hace que se repita en algunos tratados teóricos esta oposición entre el músico (teórico) y el cantor, instrumentista o compositor (práctico). Sin embargo, el verdadero propósito de algunos teóricos como Juan Bermudo y Francisco Salinas, no es el de rechazar la música práctica, considerada inferior, sino la de promover la necesidad del conocimiento teórico para el ejercicio de la música. El deseo de estos dos teóricos, según se desprende de sus escritos, es el de combatir la ignorancia de los cantores y de los instrumentistas, perjudicial para el desarrollo y enriquecimiento de la música. La influencia del pensamiento aristotélico les lleva a concebir la teoría unida a la práctica que nace de la experiencia. Para Bermudo y Salinas, es absolutamente necesario el concurso del oído en el juicio musical.

Tradicionalmente se suele presentar una oposición entre música teórica y práctica durante la Edad Media, como si las preocupaciones de los teóricos reflejadas en sus tratados fueran ajenas a la práctica musical. El punto de partida para esta oposición sería el famoso texto de Guido: «musicorum et cantorum magna est distantia...» ${ }^{1}$. Sin embargo, todas las obras teóricas de Guido

1. Guido D’Arezzo, Regulae Rythmicae, M. Gerberto, Scriptores eclesiastici de musica sacra, S. Blas, 1584, reprint, Milan, 1931, II, 25. 
tratan de problemas relativos al canto y al sistema musical ${ }^{2}$. Lo mismo puede decirse de la mayor parte de los teóricos de la Edad Media, sin olvidar que los más eruditos, añaden a las cuestiones prácticas reflexiones de carácter filosófico o moral, citas de autores clásicos, elementos de la teoría musical griega, principalmente a partir de Boecio...etc. Incluso aquellos en los que el carácter teórico o especulativo es más notable y que tratan de cuestiones eminentemente teóricas, no dejan de estar estrechamente ligados al sistema musical del que la música sonora no es más que una realización actual.

Esta oposición entre música teórica y práctica, musicus/cantor, se ha interpretado generalmente a la luz del pensamiento pitagórico-platónico, transmitido a la Edad Media por San Agustín y Boecio ${ }^{3}$.

El De musica de San Agustín (387-391), escrito en forma de diálogo, es un tratado de inspiración neoplatónica ${ }^{4}$. Concibe la música como una ciencia racional, actividad del espíritu, destacando su valor ético y su influencia en el carácter del hombre. Uno de los elementos determinantes de la presencia de la tradición neopitagórica y neoplatónica es el fundamento matemático de la música. San Agustín apoya el estudio del ritmo en las matemáticas, estableciendo el número como fundamento del orden y de la armonía. La segunda parte del primer libro esta dedicada a una introducción sobre las proporciones numéricas, su clasificación y cuáles de las proporciones matemáticas son aptas para expresar el ritmo. Las matemáticas proporcionan a la teoría musical el rigor de una ciencia, en la que la experiencia sensible debe ser transcendida con el fin de descubrir la estructura numérica escondida en los sonidos y en los movimientos. La música teórica es la ciencia que estudia la armonía y el ritmo en cuanto medidos y estructurados por los números. Una consecuencia que se desprende de esta concepción es que el oído, la experiencia sensible no es capaz de emitir un juicio universal sobre la música, sino que esto corresponde únicamente a la razón. El oído no es más que un mero intermediario que capta los sonidos y los presenta para que sean analizados por la razón ${ }^{5}$.

No obstante, aunque la ciencia de la música es considerada como especulación racional, y el conocimiento racional es superior al sensible, San Agustín concede gran importancia al oído. En frecuentes pasajes del De musica, toma como punto de partida para la reflexión un hecho de experiencia: el verso pronunciado en voz alta y captado por el oído. La audición constituye el primer grado indispensable para el juicio estético; captamos, en primer lugar el efecto agradable o desagradable de un verso por el oído, a continuación, la razón busca el fundamento matemático de esta armonía. San Agustín incluye, por tanto, la experiencia en la ciencia de la música. Este

2. Aliae regulae, G. S., II, 34; Micrologus, G. S., II, 2 ; ed. J. Smits van Waesbergher, CSM, IV, 1995, traducción inglesa por W. Babb en Hucbald, Guido, and John on Music: Three Medieval Treatises, ed. C. V. Palisca, New Haven, 1979; Epistola de ignoto cantu, G. S., II, 43.

3. Solange Corbin, «Musica spéculative et cantus pratique. Le rôle de saint Augustin dans la transmission des sciences musicales, Cahiers de Civilisation Médiévale 5 (1962), 1-12.

4. Finaert, G. et Thonnard, F. J., Dialogues philosophiques: la musique, introducción y notas, Desclée de Brouwer, Brujas, 1947.

5. «Scientia et in sola rationem esse potest». De musica $1,4,8,38$. 
aspecto constituye una innovación del tratado de San Agustín, pues la teoría no se presenta desconectada de la práctica ${ }^{6}$.

Distingue el músico del flautista o citharista, que vive de su arte, basado más en la habilidad de los dedos que en la ciencia de la música. Los tocadores de flauta y de cítara no poseen la ciencia de la música, sino que tocan en virtud de una cierta imitación. Conocen los sonidos por experiencia sensible, ligada al cuerpo y a la memoria, pero no por la ciencia que se encuentra en el espíritu. Su virtuosismo no consiste más que en la agilidad de los dedos debida al ejercicio ${ }^{7}$. Así pues, este arte, basado en la imitación, la memoria y en las disposiciones naturales de los sentidos, no es, para San Agustín, más que una forma inferior de actividad ${ }^{8}$. Se trata de una música de diversión o entretenimiento que, a los ojos de San Agustín, como es natural, es inferior a la del que cultiva la ciencia de la música además de saber tocar un instrumento.

San Agustín intenta apartar de la música todo lo que es vulgar y degradante. Sin la razón, la música no sería una ciencia noble. De aquí se desprende la mala reputación de los comediantes y músicos populares que no se conducen por la razón, sino que su fin es el placer, la gloria y el dinero ${ }^{9}$. La diferencia entre el comediante y el que posee la ciencia de la música reside en que el cómico busca un fin que se encuentra fuera de la música misma. Para San Agustín, el verdadero fin de la música es la belleza. La ciencia de la música consiste en el arte de regular bien los movimientos sonoros cuyo fin es la belleza y el encanto ${ }^{10}$.

Una concepción semejante encontramos en el De institutione musica de Boecio ${ }^{11}$. En el primer libro, capítulo IX, Boecio aborda el tema de la relación entre la razón y los sentidos en la ciencia de la música ${ }^{12}$. Aunque el punto de partida en la ciencia musical es el oído, ya que sin el fenómeno de la audición y de algo audible, no habría música, sin embargo, corresponde a la razón determinar y juzgar las consonancias ${ }^{13}$. Por otro lado, los sentidos están llenos de errores y varían de unas personas a otras, por lo que nadie que aspire a un conocimiento de la verdad puede basarse únicamente en los sentidos. Por está razón, los pitagóricos toman el oído como un mero intermediario, pero sin confíar a los sentidos el juicio definitivo. El oído puede percibir las consonancias, pero sólo la razón determina el fundamento de las consonancias.

Al final del libro I distingue tres tipos de personas relacionadas con la música: el cantor, el músico y el poeta. La cuestión planteada es la verdadera naturaleza e identidad del músico:

6. De musica 2, 11, 138 y 6, 10, 422. En la Epistola ad Memorius, 101, 3, San Agustín explica que para un buena lectura y comprensión de los cinco primeros libros, hace falta un maestro, sobre todo, para indicar por medio de la pronunciación la cantidad de las sílabas, de manera que se puede percibir por el oído las diferentes clases de ritmo.

7. Idem, 1, 4, 7-9, 40-42.

8. Marrou, H. I., Saint Augustin et la fin de la culture antique, Paris, 1938, 200.

9. De musica 1, 6, 11, 50 .

10 Idem, $1,13,28,84$.

11. Boecio, De institutione musica, Patrología Latina, Migne, LXIII, cols. 1167-1300; ed. Friedlein, Leipzig, 1867; traducción inglesa por C. Bower, Fundamentals of Music, Yale University Press, New Haven, 1989. Todas las citas de Boecio estan tomadas de la edición de Migne.

12. «Non omne judicium dandum esse sensibus». Cfr. De institutione..., I, IX, 1176.

13. Potiron, H., Boèce,théoricien de la musique grecque, Paris, 1961, 42. 
«Quid sit musicus?». En todas las artes, afirma Boecio, la razón concibe y la mano ejecuta, pero es más importante saber que hacer. Cuánto más noble, dirá Boecio, es el estudio de la música como disciplina racional que la composición y la ejecución ${ }^{14}$. La primera clase de personas dependen de los instrumentos, y están excluidos de la comprensión del conocimiento musical. Los poetas actúan movidos por un cierto instinto natural. El músico tiene la capacidad de juicio sobre ritmos y melodías. Una vez más, como en el caso de San Agustín, sólo el que conoce las leyes matemáticas que regulan la relación entre los sonidos, leyes teóricas y abstractas, merece el nombre de músico ${ }^{15}$.

El peso de la tradición en el Renacimiento hace que se repita en los tratados teóricos esta oposición o dicotomía entre el músico (teórico) y el cantor, instrumentista o compositor (práctico). Además, hay que tener en cuenta que el sistema de afinación, aceptado por los tratados teóricos, era el pitagórico en el que la cuarta es considerada consonante y las terceras y las sextas, disonantes. La práctica de la polifonía vocal e instrumental contradecía, en efecto, este sistema de afinación, que no era tenido en consideración por los músicos prácticos. Como es bien sabido, Ramos de Pareja es uno de los primeros teóricos que intenta elaborar un sistema de afinación distinto del pitagórico, en el que demuestra que las terceras y sextas son consonantes, como lo confirmará más tarde la afinación justa ${ }^{16}$. Si a esto añadimos la práctica de los distintos temperamentos mean-tone y del temperamento igual en los instrumentos, es fácil comprender las reservas o la falta de interés con la que los músicos prácticos mirarían los tratados teóricos en los que seguía vigente la tradición pitagórica.

En cualquier caso, volviendo al texto de Guido, no deja de ser desconcertante que un «cantor veterano», cambie de improviso de bando, inscribiéndose en las filas de los pitagóricos y boecianos, con toda la carga peyorativa que estos términos pueden tener en la mente de los «prácticos». En realidad, a mi modo de ver, no hay que interpretar las palabras de Guido como una mera repetición del simple cliché teóricos/prácticos que acabamos de describir. Lo que Guido pretende denunciar es la situación de los cantores ignorantes (muchos de ellos cantores por obligación), despreocupados por las reglas del canto y su correcta interpretación; sin ningún interés por mejorar su instrucción en la materia.

Es fácilmente presumible que la misma situación, de ciertos cantores ignorantes, se prolongue durante el Renacimiento, añadiéndose en este caso a los cantores, los instrumentistas que tocan de oído, que afinan su instrumento a oído y que desprecian cualquier tipo de instrucción musical ${ }^{17}$. Es cierto que siempre ha habido hombres de talento natural, capaces de

14. «Quanto igitur praeclarior est scientia musicae in cognitioni rationis, quam in opere efficiendi atque actu tantum». Cfr. De institutione..., I, XXXIV, 1195.

15. Nada más alejado de nuestra visión actual de las cosas. En el mundo moderno es el práctico, instrumentista o compositor, el que recibe el nombre de músico, mientras que el otro, el que conoce las leyes de la música, el que estudia y profundiza en la armonía, el ritmo..., en los elementos del lenguaje sonoro..., no recibe ningún apelativo particular, a lo sumo es «profesor de música» o quizá «musicólogo»...

16. Ramos de Pareja, Musica Practica, Bolonia, 1482. Goldaraz Gainza, J., Afinación y temperamento en la música occidental, Madrid, 1992, 40.

17. Bermudo, Declaración, IV, LXXV, fol. CII. 
componer, improvisar y tocar un instrumento, sin haber recibido ninguna instrucción musical, ni frecuentado ninguna institución de este tipo. Se trata, sin duda alguna, de casos excepcionales, pero no del común de los mortales que necesariamente deben pasar por el aprendizaje. Los teóricos del Renacimiento son conscientes de esta situación: un cierto número de cantores y de instrumentistas desprecian la teoría de la música, considerándola innecesaria y ajena al arte de los sonidos. Para combatir esta situación, considerada perjudicial para el progreso de la música, los teóricos adoptan, en general, dos vías:

1. La vía diáctica, poniendo el acento en la necesidad del estudio para ser un buen cantor, instrumentista... etc.

2. La vía teórica, reflexionando sobre el estatuto racional de la música como ciencia y su relación con la práctica.

En el primer caso, se encuentran los escritos de Bermudo y en el segundo, los de Francisco Salinas. El objetivo de este trabajo es mostrar el esfuerzo de ambos teóricos para combatir la ignorancia musical, intentando acercar la teoría a la práctica, en su deseo de devolver a la música el esplendor que tenía entre los antiguos.

Ambas actitudes tiene un enemigo común, el «fantasma» de la música teórica, como un mundo aparte, alejado de la práctica musical. Para ambos autores, la teórica se basa en la práctica, del mismo modo que todo conocimiento nace de la experiencia. Es necesaria, por tanto, la aportación del oído al conocimiento musical. Sin embargo, no todo conocimiento acaba en la experiencia. Es necesario también el concurso de la razón que da fundamento matemático a las relaciones entre los sonidos y que explica la verdadera razón de los fenómenos musicales.

La influencia del pensamiento aristotélico en las Universidades, concretamente en Alcalá y Salamanca ${ }^{18}$, es uno de los factores que explican la importancia dada a la unión entre razón y sentidos por nuestros dos teóricos antes citados. El aristotelismo impuso sobre todo un método de conocimiento, reconociendo tres fuentes en el saber: la autoridad, la razón y la experiencia. Estas tres fuentes están presentes en los escritos de Bermudo y de Francisco Salinas. Veamos, a continuación, cuál es la postura de cada uno de estos dos grandes teóricos respecto a la música como ciencia y su relación con la práctica.

\section{Juan Bermudo: Declaración de instrumentos musicales (1555)}

Alcalá fue uno de los bastiones del humanismo español bajo el impulso del Cardenal Cisneros, quien se inspiró directamente en la Universidad de Paris, en cuanto a los métodos de enseñanza, colación de grados y otras actividades académicas ${ }^{19}$. En esta Universidad cursó los

18. Schmitt, Ch. B., L'Introduction de la philosophie platonicienne dans l'enseignement des universités à la Renaissance, en Platon et Aristote à la Renaissance, XVIe Colloque International de Tours, París, 1976, 94. KRISTELLER, P. O., La tradizione aristotelica nel Rinascimento, Padua, 1962, 21

19. Muñoz Delgado, V., «La lógica en la Universidad de Alcalá durante la primera mitad del siglo XVI», Salmanticensis 15 (1968), 162. 
estudios de Artes Liberales Juan Bermudo, según se puede deducir de sus palabras cuando declara en el prólogo que «oyó las Matemáticas» ${ }^{20}$. Por esta época era profesor Pedro Ciruelo (1470-1554), uno de los profesores de más prestigio, por su gran erudición y cultura a juzgar por las cátedras distintas que ocupó. Ciruelo estudió Artes en Salamanca y más tarde se trasladó a París donde adquirió el grado de doctor, siendo, más tarde, profesor de matemáticas en la Sorbona. Al inaugurarse Alcalá fue nombrado profesor ocupando la cátedra tomista. En 1510 ocupó la cátedra de Teología ${ }^{21}$. Es autor de un tratado sobre las disciplinas del Quadrivium en el que se incluye la música junto a las demás ciencias matemáticas ${ }^{22}$. Uno de los libros de base para la enseñanza de la música teórica dentro del programa de Artes era el De institutione musica de Boecio.

Sin embargo, no fue la lectura de Boecio, lo que despertó el interés por la música a Bermudo, sino una circunstancia ajena a su voluntad: una larga enfermedad y su inactividad forzosa fueron la ocasión para leer una abundante literatura musical. Como él mismo declara, sus aficiones musicales permanecieron adormecidas, «enfermas» hasta que una larga enfermedad le obligó a abandonar el ejercicio de predicador, «dándose entonces a leer libros de Música». Fruto de estas lecturas serán sus escritos publicados entre 1549 y 1555: Libro primero de la Declaración de instrumentos musicales, Osuna, 1549; Arte Tripharia, Osuna, 1550, Declaración de instrumentos musicales, Osuna, 1555. Los dos primeros escritos aparecen reimpresos en la última obra.

La finalidad de los escritos de Bermudo es hacer accesible a todos los que lo desean, la ciencia de la música, teniendo como idea de fondo que el saber es un bien que ha de comunicarse. Bermudo critica duramente a los que se niegan a transmitir su saber, o una determinada habilidad, impidiendo que otros progresen en su arte. Precisamente por no ser uno de ellos afirma: «quise tomar este nuevo trabajo de poner en arte (así para el tañer, como para el modo de obrar) algunos instrumentos» ${ }^{23}$.

\section{Música teórica y práctica}

Bermudo distingue dos clases de música: inspectiva o teórica; activa o práctica ${ }^{24}$. El carácter matemático aparece como un rasgo esencial de la música teórica; es una ciencia basada en el juicio de la razón y no en el de los sentidos. En este sentido señala nuestro autor, «pertenece al músico teórico el medir y pesar las consonancias formadas en los instrumentos, no con los oídos sino con el ingenio y razón ${ }^{25}$. Cita entre los músicos teóricos a Boecio, Plutarco y a Faber Stapulense. La definición de música práctica está tomada de Guido d'Arezzo y consiste fundamentalmente en el estudio de los principios del canto.

20. Declaración, I, I, fol. I.

21. Muñoz Delgado, V., op. cit., 205.

22. Pedro Ciruelo, Cursus quatuor Mathematicarum, Alcalá, 1526.

23. Libro primero, prólogo al lector, fol., IX, v.

24. Declaración..., I, III, fol. II.

25. Ibidem. 
La música teórica es ciencia en el sentido aristotélico de conocer por causas. Este es el criterio que permite distinguir la música que es ciencia, de la que no lo es. La música que es ciencia sabe dar razón de sí misma y de los fenómenos músicales; conoce las leyes que rigen las relaciones entre los sonidos. En este sentido, Bermudo explica que existen dos modos fundamentales de conocer algo de raíz. Uno consiste en ir de la causa al efecto y el otro método sigue la vía contraria que consiste en ir del efecto a la causa. Los tañedores y los cantores conocen la música por el efecto, mientras que el teórico la conoce por la causa y razón ${ }^{26}$.

Como hemos mencionado anteriormente, Bermudo no ve una oposición entre los dos tipos de música, sino que defiende su unión. Ambas se complementan, aunque la teoría es superior a la práctica. O dicho de otro modo, la práctica debe someterse a las leyes de la razón. Para explicar la relación que une la teoría y la práctica utiliza un serie de imágenes y ejemplos: La teórica se funda en la razón que es como la reina, mientras que la práctica se ejercita con el cuerpo que es el esclavo que ejecuta ${ }^{27}$; La teórica procede del espíritu y es equivalente a él en jerarquía y rango, mientras que la práctica es inferior puesto que se encuentra a nivel de lo corporal y físico. La diferencia entre lo espiritual y lo material en el hombre introduce una reflexión moral sobre el papel de la razón como guía y gobierno del cuerpo en la conducta.

Como en el tratado de Boecio encontramos que sólo el teórico merece el nombre de músico, frente al flautista, citarista u organista:

«Aquel es dicho musico, que tiene saber para specular las proporciones musicales, los modos, y generos de musica. El que depriende a tañer sin arte, no puede ser dicho musico pues no tiene la ciencia musical: la qual no esta en la facilidad de los dedos, ni en la voz entonada: sino en el anima ${ }^{28}$.

Encontramos aquí las antiguas razones agustinianas de que el virtuosismo y la pericia en el ejecutar no hacen al verdadero músico, sino la ciencia que se encuentra en el entendimiento. Para mostrar la primacía del músico teórico sobre el práctico pone el ejemplo del capitán y los soldados en la batalla. El músico es el capitán que conoce la estrategia de la batalla y dirige la acción, los instrumentistas, en cambio, son los soldados que pelean. Otro ejemplo, tomado de Juan XXII, compara al cantor que desconoce la ciencia de la música, con el ebrio que vuelve a su casa por la fuerza de la costumbre. Los cantores que saben componer todos los modos, pero desconocen la causa de ello, el porqué, saben venir a la casa de la música, pero no saben por donde vinieron ${ }^{29}$.

Ahora bien, el propósito de Bermudo con esta discusión no es minimizar o «despreciar» el ejercico de la música práctica, sino animar a los «novicios en la música» a estudiar la teoría,

26. Declaración, I, V, fol. V.

27. Declaración, I, III, fol. III.

28. Ibidem.

29. Declaración, I, V, fol. IV. 
no conformándose con unos conocimientos elementales. «Ninguno sera musico con saber solamente la artezica del canto, aunque sin ella no lo puede ser» ${ }^{30}$.

Bermudo se lamenta del desprestigio en que es tenida la música teórica por los músicos prácticos: «Dicen los que no saben qué cosa es teórica que la práctica y la teórica son contrarias. Repugnancia y no pequeña hay en lo que dicen porque la buena práctica de la teórica nace» ${ }^{31}$.

Otras expresiones utilizadas por Bermudo para expresar la distinción entre la teoría y la práctica son las de uso y arte. El uso constituye el conjunto de conocimientos que se adquieren por experienca o dicho de otro modo, por la práctica; el arte, sin embargo es sinónimo de teoría o tratado, pues es el título común de los tratados: arte de canto llano, arte de canto de órgano... etc. Para Bermudo, no debe haber oposición entre uso y arte sino que ambos se complementan. El uso sólo sin la teoría no deja de ser algo técnico, mecánico que se repite por la fuerza de la costumbre, necesita pues del respaldo del arte, para que sea considerado música. Esta distinción de funciones da pie a una interesante discusión sobre el uso, en el sentido de práctica o experiencia, y el arte en el sentido de ciencia: «La ligereza de los dedos en los que tañen, y la facilidad de pronunciar los puntos en los que cantan: del uso y no del arte procede» ${ }^{32}$.

\section{Papel del oído en el conocimiento musical}

Bermudo apoya la necesidad del oído en el juicio musical en la filosofía de Aristóteles y el papel otorgado a la experienca sensible en el conocimiento: «Notísima cosa es que ninguna cosa hay en el entendimiento que primero no estuviese en el sentido ${ }^{33}$. Propone un ejemplo para expresar la relación entre el entendimiento y los sentidos. El entendimiento es como un mendigo que anda de puerta en puerta de los cinco sentidos mendigando: «Aquello sabe el etendimiento que oyó, rió, gustó, olió y tocó uno de los cincos sentidos» ${ }^{34}$.

Siguiendo el principio de la filosofía aristotélica, intenta combinar en el conocimiento musical ambas facultades: la razón y la experienca. De la experienca nos llega un conocimiento confuso y la razón aporta la medida exacta. El principio del juicio musical corresponde al oído, pero la perfección del conocimiento corresponde a la razón. Por otro lado, los sentidos son cambiantes y no todos los hombres tiene la misma capacidad, por ello son necesarias, para juzgar con acierto, las reglas de la razón.

Bermudo es consciente del papel del oído y del hábito perceptivo en dos temas principalmente: en el establecimiento de las consonancias y disonancias y en la aceptación de los

30. Ibidem.

31. Ibidem.

32. Declaración, I, V, fol. IV.

33. Declaración, IV, XII, fol. LXVI, v. Bermudo cita aquí el concocido principio de la filosofía aristotélico-tomista de que el entendimiento es como una tabula rasa, en la que nada hay escrito. Dicho de otra manera, sin sensación no hay conocimiento posible, no podríamos aprender nada, ni comprender. Cfr. De anima, III, 8. Summa Theologica, I, 84, 3. Probablemente se trataba de un lugar común de aceptación generalizada.

34. Declaración, IV, XII, fol. LXVI, v. 
géneros $^{35}$. La repetición en la percepción de un fenómeno hace que se convierta en algo habitual y sea aceptado sin dificultad. Esto explica que la clasificación de las consonancias varía con los tiempos. Cuando el oído se acostumbra a percibir lo que hasta entonces era considerado como disonancia, puede llegar a aceptarlo como consonancia. En este sentido afirma: «Los cantantes viejos sólo soportan la música en la que se criaron y así no sufrirán una séptima o segunda como las dan los músicos de España, porque los oídos tienen criados y cebados en la pesadumbre de la ley vieja ${ }^{36}$. Pone varios ejemplos de cambio de concepción en cuanto al valor consonante o disonante de un intervalo según la costumbre y gusto de la época. Cita en concreto el caso de la cuarta, de las terceras y de las sextas.

Bermudo encuentra también en el hábito del oído la explicación de que, aunque en los instrumentos las consonancias no sean justas, conforme a las razones matemáticas, sino temperadas, el oído acostumbrado las acepta como tales ${ }^{37}$. En realidad, no es este el motivo por el que el oído no percibe que las proporciones no son justas, sino porque la proporción de comma que se reparte entre las consonancias, según el tipo de temperamento, es tan pequeña que resulta imperceptible al oído. Sin embargo, es cierto que la repetición o el hábito interviene en la aceptación de un fenómeno, del tipo que sea. Lo que al principio puede resultar chocante acaba siendo admitido, sin dificultad, por la fuerza de la costumbre. En cualquier caso es cierto por lo que respecta a la estimación de un intervalo como consonante o disonante.

Otro factor que decide del valor consonante o disonante de un intervalo es la autoridad del que los utiliza. Cuando tal intervalo es utilizado por los buenos compositores adquiere la categoría de arte y es aceptado por la teoría como consonancia. «Y así habemos de entender el uso prevalecer y ser antepuesto al arte cuando es de varones doctos, el cual uso basta por arte ${ }^{38}$.

También se demuestra la importancia del hábito o costumbre en la aceptación del nuevo género cromático, frente al género diatónico, avalado por la tradición:

«Pues si los cantores desde las cunas en que los criaban, oyeron cantar a las moças, hermanas y madre solamente por el género diatónico y siendo moços en las calles y campos no oyeron otro, solamente han de saber este. Saben lo que oyeron y la ciencia de la música no oída no ha llegado a su entendimiento. Sufre el oído el sonido o música acostumbrada y no la inusitada. Sabe el entendiminto la música comunicada por los oídos que es la del género diatónico, ignora la que los oídos no le han comunicado que es la de los dos géneros (...). La principal parte de sonaros una música bien es tener el oído cursado a oír aquel estilo de música» ${ }^{39}$.

Sin embargo, el oído como todos los sentidos está limitado por la capacidad del que escucha y está sujeto a error, por ello tiene que someterse al juicio de la razón ${ }^{40}$. En conclusión,

35. Este tema es desarrollado en el libro IV.

36. Declaración, IV, XII, fol. LXVI.

37. Declaración, IV, XI, fol. LXV.

38. Declaración, IV, XII, fol. LXVI.

39. Declaración, IV, XII, fol. LXVI.

40. Declaración, IV, XCIII, fol. CXIII. 
según Bermudo, hay que tomar los oídos de los buenos músicos por nivel y regla de las consonacias y no los de los principiantes, teniendo en cuenta que cuando uno se acostumbra a oír un determinado intervalo, le suena bien. El oído de los principiantes se puede comparar, dice Bermudo, a la conciencia de los pecadores.

«Tanto tiempo puede uno sufrir pecados pequeños que venga no tan solo a no remorderle la conciencia sino a defender los pecados. Los principiantes que el oído hecho con canto de bárbaros, no corregido por sabio maestro y por sí solamente ejercitado propinquos están a este error. Cásense pues con las reglas de los verdaderos músicos, no estén amancebados con sus oídos y depriendan de los excelentes varones y serán libres de tan grande error» ${ }^{41}$.

\section{Necesidad de instrucción para ser un buen instrumentista: el recurso a la autoridad}

Bermudo defiende la necesidad de acudir a los autores clásicos ya que todo conocimiento progresa a partir de lo ya adquirido. Así, anima a los instrumentistas a conocer lo que los músicos anteriores han descubierto: «Teniendo los tañedores en mucho los que los antepasados han descubierto, así en hallar instrumentos, como en primores musicales y queriendo trabajar, pasarán adelante todos ellos» ${ }^{42}$. Manifiesta una cierta actitud humilde, que contrasta con la actitud adoptada en otras ocasiones, al reconocer que nadie puede creerse en posesión de todo el conocimiento. Por ello, el estudio de los autores antiguos es necesario, aunque requiera largo tiempo y esfuerzo:

«Confieso verdad que ha ocho años bien complidos que principalmente estoy ocupado en estudiar los dichos autores y que me pone en admiración lo mucho bueno que en música nos dejaron. Lo cual antes del dicho tiempo, aunque me tenía por práctico y teórico, no sabía, ni había visto, ni pensaba tales cosas estar escritas ${ }^{43}$.

A los que quieren iniciarse en la composición de canto llano y canto de órgano aconseja, en primer lugar, aprender de buenos maestros y en segundo lugar, imitar a los buenos músicos. Con gran sentido común comenta que el que aprende a tañer de mal maestro quedará simpre con mal aire; del mismo modo el que empieza a componer imitando mala música, difícilmente mejorará en el arte de componer. Bermudo sugiere comenzar por el repertorio del canto llano clásico: el canto gregoriano, el toledano y el ambrosiano:

«Los que quisieren atinadamente componer, miren los autores ya dichos y con su buen entendimiento y ejercicio sabrán elegir las flores de la música antigua y descubrirá más música en un mes que por sí sin maestros alcanzará en un año. Aunque todos los autores y otros muchos más vea elija y tenga alguno particular que más le contente por familiar» ${ }^{44}$.

41. Declaración, IV, XCIII, fol. CXIII.

42. Declaración, IV, LXXXV, fol. CIX.

43. Declaración, IV, LXXXV, fol. CIX.

44. Declaración, V, IX, fol. CXXIV, v. 
Cuando no se tiene un buen maestro hay que buscar en buenos libros que traten de la materia. Otra manera de aprender consiste en estudiar las obras de músicos excelentes. «Ningún hombre sabio puede decir tanto por letras cuanto descubrirá en la composición». Estudiando las obras de los grandes compositores se aprende el modo de poner las consonancias y la manera de hacer las cláusulas. Además se pueden ver «novedades, primores y todo dechado de buena música» ${ }^{45}$.

Según Bermudo, uno de los motivos por los que se ha perdido la honestidad de la música es la falta de instrucción teórica. Bermudo se dirige principalmente a los que no tienen una formación teórica y desprecian la instrucción científica. Para ellos, hace saber que la música antigua tenía grandes cualidades y que los músicos de ahora han intentado resucitar parte de esta música perdida. «Grandes son los primores que en este tiempo se usan en la música: pero no son tan nuevos que no los hallemos en la musica de los antiguos» ${ }^{46}$. Con un cierto tono nostálgico defiende la excelencia de la música antigua:

«Los que desconocen las grandezas de la música antigua y sus efectos piensan que la música actual se encuentra en la cumbre de la perfección, pero los que tienen noticia de la otra, lloran por la perdida grande» ${ }^{47}$.

Para probar sus razones aduce que ningún músico de nuestros tiempos ha conseguido los efectos y excelencias que hicieron los antiguos. En la misma línea de comparación entre la música antigua y la moderna, señala Bermudo la dedicación al estudio y al ejercicio de los músicos antiguos, mientras que los modernos confían más en la habilidad espontánea o natural. En este sentido observa que mientras los discípulos de Pitágoras tocaban todas las noches la vihuela, pocos músicos de España han oido la música en el estudio general ${ }^{48}$.

\section{Carácter pedagógico}

El carácter pedagógico de la Declaración es uno de los aspectos más llamativos y puesto de relieve por su autor. Bermudo otorga una gran importancia a la transmisión del saber, como sugiere la famosa frase de Aristóteles al inicio de la Metafísica: con la que comienza nuestro autor la Declaración:

«... todos los hombres desean saber. Si algunos quedan ignorantes no es por falta del deseo natural sino o porque no quieren trabajar o porque carecen de buenos maestros o porque los maestros no quieren enseñar» ${ }^{49}$.

45. Declaración, V, IX, fol. CXXIV, v.

46. Declaración, I, XIII, fol. XII.

47. Declaración, I, XIII, fol. XII.

48. Declaración, I, XIV, fol. XIII.

49. Declaración, prólogo primero para el piadoso lector. 
Denuncia el hecho de que en España hay excelentes vihuelistas y tañedores de órgano que no quieren comunicar su saber. Como consecuencia, a pesar de haber «tan grandes ingenios, $\tan$ delicados juicios, tan inventivos entendimientos, restan todas las artes muertas» ${ }^{50}$. Bermudo alude a ciertos vihuelistas que cuando tañen no quieren que se les vea la postura de las manos para que no se la copien. Achaca a la falta de buenos maestros los errores y desviaciones en el canto: «Los malos tañedores que tocan en un modo cuando el coro canta en otro es por culpa de los maestros». Por todos estos motivos -la falta de instrucción de los cantores y tañedores-, Bermudo decide escribir sus libros, para que todo el que quiera aprender a tañer un instrumento, tenga los conocimientos necesarios para poder hacerlo ${ }^{51}$.

Otra manifestación del interés pedagógico de Bermudo son los consejos y avisos que a lo largo de su obra da a los tañedores, cantantes y a los que dirigen el coro. Así, en el libro IV de la Declaración dedica el primer capítulo a una serie de consejos de carácter moral sobre la necesidad del estudio y otros, de carácter práctico para los principiantes, deseosos de aprender a tañer el monachordio. Bermudo predica la constancia y el esfuerzo como remedio para superar los obstáculos y dificultades que se pueden presentar en el aprendizaje de un instrumento. «No hay cosa tan recia y dura que la continuación y trabajo no ablande y cave, ni tan dificultosa, que la perseverancia no haga fácil» ${ }^{52}$. Reflexiones de tipo pedagógico aparecen constantemente a lo largo de la obra, incitando al lector a asimilar el contenido de los libros, aplicando el entendimiento ${ }^{53}$.

En definitiva, toda la parte teórica desarollada en los libros I, III y V constituyen el bagage teórico que todo músico, organista vihuelista o director de coro debe poseer para ser un buen músico.

\section{Francisco Salinas: De musica libri septem (1577)}

Una parte importante de la actividad musical del Maestro Salinas se desarrolla en el entorno intelectual de la Universidad de Salamanca. Primero como estudiante, frecuentó las aulas de griego, artes liberales y filosofía. No sabemos cuánto tiempo pasó Salinas en Salamanca, pero es probable que estuviera tres o cuatro años, tiempo que duraban los estudios de la facultad de artes, incluyendo además de las materias propias del Trivium y el Quadrivium, la filosofía natural y la filosofía moral, ambas explicadas a partir de Aristóteles. Dentro de la filosofía natural se estudiaban la Metafísica, los Problemas, la Física y el De anima. Los textos para la filosofía moral eran las dos Eticas de Aristóteles y la Política ${ }^{54}$. La formación filosófica y científica era,

50. Ibidem.

51. Libro primero, fol. V.

52. Declaración, IV, I, fol. LX.

53. Declaración, IV, LXXII, fol. XCX, a.

54. Valero García, P., La Universidad de Salamanca en la época de Carlos V, Salamanca, 1988, 267. 
por tanto, marcadamente aristotélica y su influencia se hará sentir en el concepto de ciencia que servirá de modelo a Salinas para escribir los Siete libros sobre Música.

Pasados unos 30 años, aproximadamente, vuelve a Salamanca como catedrático de música en 1567. Permanecerá como profesor hasta su jubilación en $1587^{55}$. En este contexto de enseñanza universitaria, verán la luz los Siete libros sobre música, impresos en 1577 que Salinas escribió, probablemente, como texto para el desarrollo y explicación de sus clases.

Nuestro autor es consciente de la distancia que separa los tratados especulativos de inspiración boeciana de la práctica musical. Asumiendo que no hay ciencia posible que no lo sea de los hechos concretos que estudia, Salinas intentará tender un puente entre el conjunto de teorías de carácter especulativo sobre la armonía y el ritmo y la realización concreta de estos principio teóricos en la práctica musical y poética. De su estudio afirma:

«En él vamos a demostrar a los hombres de nuestro tiempo de dónde han salido las numerosas y variadas doctrinas de aquellos que sobre música han escrito. De esa manera esperamos reconciliar y aúnar a los que gozan de la práctica de la música con los que teorizan sobre ella» ${ }^{56}$.

\section{La música como ciencia}

Para la filosofía aristotélica todo conocimiento humano deriva a través de la representación y la abstracción de la percepción sensible ${ }^{57}$. El recurso a la experiencia permitirá a Salinas recabar el estatuto de la música como ciencia, según el modelo aristotélico.

Los años pasados como estudiante en la Universidad de Salamanca, así como la deuda con el tratado de Tolomeo explican la influencia del aristotelismo en el De musica libri septem. La noción de ciencia como saber universal, inmutable y necesario y la elaboración del método científico constituyen la contribución más importante de Aristóteles a la teoría musical de Salinas. Ambos aspectos están presentes en su obra, donde aparece claramente descrito el modelo de ciencia que conduce a la verdad sin error, pero apoyándose siempre en la experiencia, en el uso de la práctica y no sólo en el razonamiento abstracto. Salinas destaca la conexión entre ciencia y verdad cuando dice: «La verdad es la única razón de la investigación» ${ }^{58}$. Y en otro momento añade: «la verdad brilla por sí misma y a todos los que la buscan se muestra por igual» ${ }^{59}$. A pesar de estas afirmaciones, Salinas es consciente de la dificultad que existe a veces para aceptar la verdad cuando se ha vivido en el error: «No es fácil persuadir al vulgo porque

391.

55. Esperabe Arteaga, E., Historia pragmática e interna de la Universidad de Salamanca, II, Salamanca, 1917,

56. Salinas, F., De musica libri septem, Salamanca, 1577, facsímil por M. S. Kastner, Kassel, 1958, traducción española por I. Fernández de la CuEsta, Siete libros sobre música, ed. Alpuerto, Madrid, 1983, I, I, 36. Para las referencias de Salinas hemos utilizado la edición española.

57. Kristeller, P. O., La tradizione..., 22.

58. Siete libros..., IV, 408.

59. Idem, IV, 400.

60. Idem, VII, III, 665. 
tanta es la fuerza de la costumbre que cuando ha arraigado por una idea falsa, nada hay más opuesto a recibir la verdad» ${ }^{60}$.

Siguiendo el método trazado por Aristóteles, insiste en la unión de la razón y los sentidos en el proceso del conocimiento. Estas dos facultades actúan conjuntamente. Una ciencia construida solamente según las leyes de la lógica y sin tener en cuenta la experiencia nos llevaría a un conjunto de teorías inútiles, absolutamente desconectadas de la realidad. Es el caso de las doctrinas pitagóricas y de Tolomeo cuando establecen afirmaciones que contradicen la realidad sensible. En el lado opuesto, tenemos la posición de los prácticos que tienen un conocimiento ligado a la experiencia de su trabajo, pero no saben dar razón de lo que practican. Es decir, su conocimiento es un conocimiento fenoménico y no de las causas. A lo largo de toda la obra, Salinas mantiene que la ciencia se construye a partir de los datos de experiencia. Los datos aportados en la percepción sensible constituyen el material que la razón analiza, ordena y clasifica.

La labor del científico es ordenar y clasificar el contenido de la experiencia. La clasificación constituye una disposición ordenada de objetos en un conjunto mientras que la explicación descompone en partes el material aportado en la experiencia, estableciendo una distinción entre el todo y las partes ${ }^{61}$. Estas dos propiedades de la ciencia están presentes también en el tratado de Salinas. De modo habitual el objeto de estudio es situado en su contexto, utilizando divisiones y subdivisiones, así como esquemas que ayudan a comprender la estructura lógica y la relación entre los distintos elementos de estudio. En esto consiste, para Salinas la tarea del científico: «Aquél a quien pertenece el ordenar (Aristóteles dice que pertenece al sabio) también le pertenecen conocer el ordenar y decidir respecto de dicho orden ${ }^{62}$. El orden habitual es comenzar por la definición para pasar después a la división. En ocasiones la definición consiste en una aclaración de la acepción del término empleado y casi siempre Salinas precisa el uso y sentido de los términos utilizados.

Siguiendo a Aristóteles, concibe la música como una ciencia teórica que ocupa una posición intermedia entre la física y las matemáticas. Todo el libro I se dedica a exponer el fundamento matemático de la ciencia armónica. Utiliza demostraciones matemáticas para demostrar las proporciones de las consonancias e intervalos y rechaza por la misma vía racional las argumentaciones de los pitagóricos. Distingue en la armónica el elemento sonoro y el numérico, aunque ambos están indisolublemente unidos dentro de la misma ciencia. Por el estudio del sonido, la música goza del mismo estatuto que la física. Sin embargo, no es el sonido en cuanto realidad física lo que interesa al músico. Por la aplicación de los números y las proporciones al sonido, la música es una disciplina matemática. Con todo, insiste Salinas, el objeto de estudio de la música no es el sonido, ni el número, sino el número sonoro. Transcribimos a este propósito las palabras del propio Salinas:

61. Le Blond, J. M., Logique et méthode chez Aristote, Paris, 1970, 292.

62. Siete libros..., V, III, 418. 
«La música no es propiamente matemática, ni física, sino algo entre medio. Si nos fijamos en los sonidos, es física, por cuanto el sonido es algo material y móvil, y esto pertenece a la física. Pero en cambio el número, esto es, las relaciones numéricas que pueden endenderse muy bien fuera de la materia y del movimiento, pertenecen a las matemáticas» ${ }^{63}$.

El sonido es la parte material de la música en cuanto mensurado y el número es la parte formal en cuanto mensurante, siendo la forma matemática más noble que la materialidad del sonido en $\mathrm{si}^{64}$. Por este motivo la matemática constituye el fundamento de la doctrina musical pues sin el número, tanto simple como en relación con otros «toda la doctrina de la música se vendría abajo» ${ }^{65}$.

\section{Razón y sentidos}

El capítulo III del libro I expone la necesidad de los sentidos y de la razón dentro de la armónica. «Las facultades o jueces que intervienen en la armónica son los sentidos y la razón. pero no de la misma manera» ${ }^{66}$. Aquí Salinas cita directamente a Tolomeo aunque los conceptos que permiten articular el discurso son los conceptos aristotélicos de materia y forma. Según Tolomeo, el sentido juzga la materia y el efecto; la razón, la forma y la causa ${ }^{67}$. De esta afirmación se desprende que lo que es la forma con respecto a la materia, es decir, su perfección y acabamiento, así es la razón para los séntidos:

«Los sentidos captan lo más cercano a ellos, y sólo la razón capta la integridad del objeto. La razón necesita que el sentido proporcione la sensación material y próxima a él para poder captar dicha totalidad. Así pues, el sentido conoce de manera confusa la materia fluida e inestable - en el caso de la música - y la razón conoce esto mismo despojado de su misma materia con toda exactitud y realidad» ${ }^{68}$.

Este texto en el que Salinas parafrasea a Tolomeo, presenta una gran semejanza con aquel otro de Aristóteles en el libro I de la Física, cuando afirma que el camino natural hacia la ciencia es ir de lo más conocido para nosotros y más claro a lo que es más claro en sí mismo y más cognoscible. Aristóteles advierte que lo que para nosotros es manifiesto y claro es lo que está más entremezclado. Sólo después por la vía del análisis podemos desgajar los principios y los elementos. Por esto hay que ir de lo general a lo particular, pues el todo es más cognoscible por sensación y lo general es como un todo: encierra una pluralidad que constituye como su parte ${ }^{69}$.

63. Siete libros..., I, IV, 40.

64. Idem, I, IV, 40.

65. Idem, I, IV, 41.

66. Siete libros..., I, III, 38.

67. Tolomeo, Armónica, I, 1, ed. BARKer, Greek Musical Writings, II, Cambridge, 1989, 276.

68. Siete libros..., I, III, 39.

69. Aristóteles, Physique, 184 a, 16-26. Traducción y notas por H. Carteron, Les Belles Lettres, Paris, 1926. 
En este texto, comenta A. Bélis, lo general designa la percepción confusa que es general porque es indistinta y en ella se da todo mezclado. El análisis de la percepción en el que se da el todo confuso y mezclado permite descubrir la unidad, el todo organizado en la composición de las partes, unidad que es imperceptible en la sensación ${ }^{70}$

Salinas afirma que tan necesario es el oído para la música como la razón. Ambos søn absolutamente imprescindibles ${ }^{71}$. El oído capta los sonidos y la razón la relación armónica. La labor de los sentidos es previa en la mayoría de los casos a la razón. El oído precede a la razón, puesto que su labor consiste en aportar los materiales. Por ello, afirma Salinas, «la intervención del oído es de absoluta necesidad $»^{72}$. La necesidad del oído se muestra especialmente a la hora de establecer las consonancias. «Las consonancias, advierte Salinas, son mucho más fáciles de percibir por los sentidos que los restantes intervalos menores, por tener una medida más proporcionada». A continuación apoya esta afirmación en un principio establecido por Tolomeo, de que «cuanto más proporcionada es una medida tanto más perceptible es» ${ }^{73}$. Esto prueba una vez más que no puede haber contradicción entre los sentidos y la razón, habiendo una maravillosa armonía entre ambos. Sin embargo, no basta con el oído, porque, de no acudir en su auxilio la razón, quedaría manco y débil: «El método para establecer las consonancias y los intervalos menores utiliza la experiencia y el uso de la música práctica y después aplica la razón para establecer el cálculo matemático, pero nunca se deja guiar por la razón en contra de la experiencia» ${ }^{74}$. La experiencia en Salinas, como en Aristóteles es sobre todo familiaridad con los fenómenos, experiencia personal que continua la tradición de los antiguos ${ }^{75}$.

Tampoco la razón se basta a sí misma pues no puede juzgar, si no entra por las ventanas de los sentidos lo que debe ser conocido y juzgado. La función de la razón es analizar, juzgar y apreciar las minuciosas y sutiles diferencias que jamás el sentido podría distinguir Para mayor precisión en la delimitación de las funciones de la razón y los sentidos Salinas establece el paralelismo entre el objeto y la facultad correspondiente. Así, los sentidos captan la materia que es el sonido y la razón las proporciones numéricas que miden los sonidos ${ }^{76}$.

Apoyándose en el oído y en la práctica de la música, Salinas admite las $3^{\text {as }}$ mayor y menor como consonantes. El oído capta que son consonantes y la razón muestra sus proporciones superparticulares $5 / 4$ y $6 / 5$ respectivamente. Comenta Salinas que si los pitagóricos no se equivocaron al establecer las consonancias hasta la $4^{a}$ es debido a que usaron de la razón y de la experiencia. «Sin embargo al establecer los intervalos menores tan sólo aplicó (Pitágoras) el juicio de la razón, sin tener en cuenta los datos del oído» ${ }^{77}$

70. Belis, A., Aristoxène de Tarente et Aristote, le traité d'harmonique, Paris, 1904, 36.

71. Siete libros..., I, III, 38.

72. Idem, I, III, 38.

73. Idem, II, IV, 116.

74. Idem, II, XI, 131.

75. LE BLOND, op. cit., 433.

76. Siete libros..., I, III, 38.

77. Idem, II, X, 127. 
Ahora bien, la ciencia no se queda sólo en el testimonio de los sentidos. Hace falta el concurso de la razón que ilumina el material aportado por los sentidos y lo hace inteligible. La razón examina y aporta el juicio definitivo, la razón exacta de esta conveniencia. «Tal debe ser la intención del músico armónico: mostrar conforme a la razón lo que aparece claro a los sentidos para todas las gentes. Las teorías que (...) construye, deben ser de tal naturaleza, que lo que en ellas se afirma esté conforme con la razón y los sentidos» ${ }^{78}$. Además la razón proporciona la seguridad en la certeza.

«No hay nada más cierto y más seguro que las proporciones de los intervalos y la numerabilidad de los tiempos, si del jucio, a veces falaz e inconstante, de los oídos se pasa al examen seguro de la razón» ${ }^{79}$.

En otras ocasiones, la experiencia no precede al juicio de la razón sino que lo confirma o bien puede rechazarlo. Así al hablar del archicémbalo, instrumento diseñado por N. Vicentino ${ }^{80}$, Salinas lo critica porque las consonancias no son justas en este instrumento. «Siempre lo he comprobado por la experiencia, cuantas veces he querido temperarlo según la teoría de este autor he experimentado en las consonancias una imperfección tan grande que los oídos no lo podían soportar» ${ }^{81}$. Los ejemplos musicales utilizados por Salinas sirven de confirmación a las teorías expuestas. Es el caso de la Misa de Juan Dumont de tres bemoles, (si b, la b, mi b) citada para probar el uso del género enarmónico ${ }^{82}$. Igualmente después de exponer los argumentos presentados en favor del la cuarta como consonancia y de rebatir las opiniones de algunos músicos prácticos que lo colocan entre las disonancias, aduce algunos ejemplos que testimonian su uso como consonancia. Este procedimiento aparece explícitamente descrito por Salinas:

«Podemos realizar la demostración de tres maneras: primeramente, por el testimonio de los hombres más sabios y beneméritos de la música; en segundo lugar, por la misma razón, que vale más, como dice San Agustín, que el propio testimonio de los hombres; y por fin, valiéndonos de ejemplos de los mejores compositores» ${ }^{83}$.

Entre otros ejemplos cita la afinación de las cuerdas en las vihuelas y laúdes por cuartas, los cantos populares de los griegos en la iglesia y, sobre todo, el Resurrexit de la Misa del $6^{\circ}$ tono l'homme armé de Josquin des Près, que comienza por un iniervalo de cuarta ${ }^{84}$.

78. Idem, IV, XVII, 365.

79. Idem, V, III, 418. KASSEL, 1959.

80. Nicola VICENTINo, L'antica musica ridotta alla moderna prattica, Roma, 1555, facsímil por E. LOXINSKY,

81. Siete libros, III, XXVII, 300.

82. Idem, III, VIII, 238.

83. Idem, II, IX, 125.

84. Idem, II, IX, 126.

85. Idem, $\mathrm{V}, \mathrm{XV}, 460$. 
En la teoría rítmica la aceptación del anfíbraco se basa en su efecto rítmico agradable al oído, como lo prueban los fragmentos musicales presentados por Salinas ${ }^{85}$. Los ejemplos constituyen la verificación constante de la doctrina expuesta. El ejemplo sustituye en muchos casos la explicación, pues, dice Salinas, imitando a Séneca:

«... según dice Séneca, las explicaciones son largas y difíciles y, en cambio, un ejemplo es más breve y eficaz» ${ }^{86}$.

Otra manifestación de cómo la razón y la experiencia actúan en conjunto es la crítica a la formación de los intervalos menores de la doctrina pitagórica. Salinas rechaza el semitono menor pitagórico o limma 256/243 porque es totalmente inepto para la armonía. Esto lo prueba por el triple camino de la autoridad, la razón y la experiencia: en primer lugar, Tolomeo demuestra que es un intervalo incóncino ${ }^{87}$. En segundo lugar, la razón hace ver que se trata de una proporción superpartiens; por último, la experiencia demuestra que es un intervalo imposible de cantar ${ }^{88}$.

En conclusión, podemos ver que el tratado de Salinas está totalmente impregnado de la enseñanza de Aristóteles por su vocabulario, por los principios filosóficos y por la teoría de la ciencia, los instrumentos de conocimiento y el método utilizados por el músico de Burgos. La música se presenta en la obra de F. Salinas como una ciencia, cuyos conocimientos se expresan en proposiciones verdaderas que proporcionan la razón exacta de los fenómenos musicales. Los instrumentos para elaborar el conocimiento científico son la razón unida a la experiencia. El recurso constante a la experiencia a lo largo de los Siete libros desmarca a Salinas de la corriente pitagórica, poniendo de manifiesto su concepción aristotélica de la ciencia. Este método de conocimiento que se inicia en la percepción sensible, es completado por la contrastación con las teorías de otros autores. En definitiva, la ambición de Salinas es la de abarcar en un saber científico todo lo que se puede decir y conocer de una actividad humana, bajo todos los aspectos. En suma, no ignorar nada de lo que tiene que ver con la ciencia de la música. Como él mismo declara en el prólogo, el De musica libri septem intenta reunir todo lo que de armonía y de ritmo debe saber el músico ${ }^{89}$.

86. Idem, I, XV, 62 .

87. Armónica, I, 10.

88. Siete libros..., II, XVIII, 156 y ss.

89. Idem, prólogo, 30 . 